\title{
A Comprehensive Model for the Simulation of Reinforced Concrete Deterioration due to Corrosion
}

\author{
Xingji Zhu, Zhaozheng Meng* \\ Harbin Institute of Technology at Weihai, Department of Civil Engineering, Weihai, China \\ 161210228@stu.hit.edu.cn
}

Keywords: carbonation, chloride transport, oxygen transport, corrosion reaction, electro-chemical process

Abstract: A comprehensive model of steel reinforced concrete corrosion process is established. The coupled effect of carbonation, chloride transport and oxygen transport are discussed in this paper. Mathematical formulae depicting mass transport in concrete structures are given to simulate the mass transport process. This paper also introduces corrosion distribution and reaction process of concrete structures.

\section{Introduction}

Recent years have witnessed a wide application of concrete material, because of its construction convenience, high strength and relatively long service life. However, reinforced concrete structure has suffered corrosion problems due to aggressive agents from de-icing salts and marine moisture [1]. In addition, corrosion process will impair the carrying capacity of rebars set in the concrete $[2,3]$, and the concrete also suffer spalling and cracking due do the volume expansion of rust layer. However, repairmen and replacement of building components are expensive. Consequently, to predict the crack penetration and service life span more precisely, studies focusing on the corrosion process are attached the utmost importance.

The corrosion of reinforced concrete structure is a complicated process, which involves carbonation and transport of chloride ions, moisture and oxygen. Coupled processes are also included in the corrosion process. It is known that concrete is an alkaline material [1], so a passive layer is generated on the surface of rebars. However, when the $\mathrm{pH}$ value decreases below 11 or the chloride content reaches a threshold, the depassivation process initiates. Rebars are exposed to aggressive agents. Therefore, the microcell corrosion process begins. On the other hand, cracks can accelerate the diffusion process of aggressive agents, which leads to more corrosion products and cracks penetration $[4,5]$.

It is notable that the volume of corrosion product is 3 to 6 times larger than the original volume of steel reinforcement [6], so the concrete cover undertakes extra pressure. Once the corrosion induced stress exceeds the limiting stress, crack initiates.

\section{Mechanism of mass transport in concrete}

\subsection{Carbonation process}

Numerous researchers have focused on the carbonation process concrete material. Here, the governing equations concerning the carbonation process are given by

$$
\frac{\partial\left(\mu-\mu_{w}\right) C_{c}}{\partial t}=\left(\mu-\mu_{w}\right) D_{c} \nabla^{2} C_{c}-Q_{c h}-Q_{C S H}
$$

where $\mu$ is porosity varying with carbonation degrees; $\mu_{w}$ is the volume fraction of evaporable pore water; $C_{c}$ is the molar concentration of carbon dioxide in pores of concrete; $D_{c}$ is the diffusion coefficient of gaseous carbon dioxide in concrete; $Q_{c h}$ is the rate of reaction between carbonation and calcium hydroxide; $\mathrm{Q}_{\mathrm{CSH}}$ is the rate of reaction between carbonation and CSH $\left(3 \mathrm{CaO} \cdot 2 \mathrm{SiO}_{2} \cdot 3 \mathrm{H}_{2} \mathrm{O}\right)$. It is worth noting that the determination of all parameters mentioned above 
can be found in literature [7]

\subsection{Chloride transport in concrete}

Three kinds of chloride are discovered in the concrete material, which are chloride ions in pore solution in concrete, bond chloride and chloride salt that has the potential to be turned into chloride ions by carbonation process. Based on the impact of carbonation on chloride transport, the formula depicting free chloride transport in concrete can be given

$$
\frac{\partial \mu_{w} C_{f c}}{\partial t}=\nabla \cdot\left(D_{f c}^{c a r} \nabla \mu_{w} C_{f c}\right)+\nabla \cdot\left(\mu_{w} C_{f c} D_{h}^{c a r} \nabla h\right)+Q_{r c}
$$

where $C_{f c}$ is the content of chloride ions in concrete pore solution in concrete; $D_{f c}^{c a r}$ is the diffusion coefficient of chloride ions with the consideration of carbonation; $D_{h}^{c a r}$ is the diffusion coefficient of water with the consideration of carbonation, which is given by the following equations; $Q_{r c}$ is the conversion rate from bond chloride to free chloride because of the carbonation process, and $Q_{r c}$ can be determined in literature [8]. As for $D_{f c}^{c a r}$ and $D_{h}^{c a r}$, they can be determined by equations given in literature [8] also.

\subsection{Oxygen transport in concrete}

It is until the beginning of electrochemical process that the oxygen participate in the transport process in concrete. Hence, the oxygen transport process can be simplified, and the governing equation with the consideration of convection can be drawn by

$$
\frac{\partial C_{o}}{\partial t}=\nabla \cdot\left(D_{o}^{c a r} \nabla C_{o}\right)+\nabla \cdot\left(C_{o} D_{h}^{c a r} \nabla h\right)
$$

where $C_{o}$ is the molar concentration of oxygen that has been dissolved in porous water in concrete, and $D_{o}^{c a r}$ is the diffusion coefficient of dissolved oxygen with the consideration of carbonation degrees, which can be referred in literature [7].

\section{Mechanism of corrosion reaction}

\subsection{Corrosion current density}

It is notable that there are two forms of corrosion when corrosion process initiates. The first one is microcell corrosion on the active zone, and the second one is macrocell corrosion on the area between active zone and passive zone. Additionally, the process of electrochemical reactions can be illustrated by the following chemical equations:

$$
\begin{gathered}
\mathrm{Fe} \rightarrow \mathrm{Fe}^{2+}+2 \mathrm{e}^{-} \\
\mathrm{O}_{2}+2 \mathrm{H}_{2} \mathrm{O}+4 \mathrm{e}^{-} \rightarrow 4 \mathrm{OH}^{-} \\
\mathrm{Fe}^{2+}+2 \mathrm{OH}^{-} \rightarrow \mathrm{Fe}(\mathrm{OH})_{2}
\end{gathered}
$$

It is notable that the cathodic reaction (the reduction reaction of oxygen) is not limited to active zone, and there is cathodic reaction on passive zones due to the microcell corrosion. Moreover, the corrosion products are not limited to $\mathrm{Fe}(\mathrm{OH})_{2}$, but we regard the ferric hydroxide as the corrosion product here, for the sake of simplicity.

The current density of macrocell corrosion can be drawn as

$$
\begin{aligned}
& j_{\text {mac }}^{a}=j_{\text {and }}^{a}-j_{\text {ctd }}^{a} \\
& j_{\text {mac }}^{p}=j_{\text {and }}^{p}-j_{\text {ctd }}^{p}
\end{aligned}
$$

where $j_{m a c}^{a}$ and $j_{m a c}^{p}$ are current density on active zones and passive zones respectively; $j_{\text {and }}^{a}$ and $j_{c t d}^{a}$ are the anodic and cathodic current density on active zones respectively; $j_{\text {and }}^{p}$ and $j_{c t d}^{p}$ are the anodic and cathodic current density on passive zones respectively, and $j_{\text {and }}^{p}$ can be considered as 0 according to literature [9]

As for the current density of microcell corrosion, according to literature [10], the current density on active zone $j_{m i c}^{a}$ equals the cathodic current density on active zones $j_{c t d}^{a}$. Therefore, the total current density can be given by 


$$
j_{\text {tot }}^{a}=j_{\text {mic }}^{a}+j_{\text {mac }}^{a}=j_{\text {ctd }}^{a}+j_{\text {and }}^{a}-j_{\text {ctd }}^{a}=j_{\text {and }}^{a}
$$

where the determination of relating parameters $j_{\text {and }}^{a}, j_{\text {ctd }}^{a}$ and $j_{\text {ctd }}^{p}$ can be found in literature[10].

\subsection{Electric potential and polarization of steel reinforcement}

Here, we employ a Laplace equation to express the electrical potential $\phi$ in concrete, which can be drawn as

$$
\nabla \cdot\left(\frac{1}{R} \nabla \Phi\right)=0
$$

where $R$ is the electrical resistance, which can be determined by the properties of concrete and the water saturation.

On the contact surface between rebars and concrete, the macrocell current densities of active and passive zones are equal to the current densities which flows on the anodic and cathodic area. Consequently, the boundary condition of electrical potential can be calculated. The electrical potential on the rebars can be given by

$$
j_{\text {mac }}^{a} g_{a}\left(C_{f c}, p H\right)+j_{\text {mac }}^{p} g_{p}\left(C_{f c}, p H\right)=-\left.\mathbf{n} \cdot\left(\frac{1}{R} \nabla \Phi^{r}\right)\right|_{\text {interface }}
$$

where $\mathbf{n}$ is the unit vector which is perpendicular to the contact surface, and $g_{a}\left(C_{f c}, p H\right)$ and $g_{p}\left(C_{f c}, p H\right)$ are switch functions which can be depicted by

$$
\begin{aligned}
& g_{a}\left(C_{f c}, p H\right)=\left\{\begin{array}{lllll}
0 & \text { for } & 0 \leq \frac{\phi_{w e} C_{f c}}{b}<C_{t h}^{c a r} & \text { and } & p H>p H_{t h} \\
1 & \text { for } & \frac{\phi_{w e} C_{f c}}{b} \geq C_{t h}^{c a r} & \text { or } & p H \leq p H_{t h}
\end{array}\right. \\
& g_{p}\left(C_{f c}, p H\right)=\left\{\begin{array}{lllll}
1 & \text { for } & 0 \leq \frac{\phi_{w e} C_{f c}}{b}<C_{t h}^{c a r} & \text { and } & p H>p H_{t h} \\
0 & \text { for } & \frac{\phi_{w e} C_{f c}}{b} \geq C_{t h}^{c a r} & \text { or } & p H \leq p H_{t h}
\end{array}\right.
\end{aligned}
$$

where $p H$ means $\mathrm{pH}$ value of the porous solution in the concrete; $C_{t h}^{c a r}$ is the threshold of chloride ions content; $p H_{t h}$ is the threshold of $\mathrm{pH}$ value when depassivation process initiates; $b$ is the binder content percentage in concrete; $\varphi_{w e}$ is the volume fraction of porous water that can evaporate. More detailed equations determining these above-mentioned parameters can be found in literature [8]

\section{Corrosion distribution}

Based on the mass transport equations mentioned above and the electrochemical reactions mentioned in literature [7], we can add up total current density at each zone on rebars. According to the Faraday law, the radius reduction of rebars on radial direction can be given by

$$
u_{\text {red }}(\theta, t)=\frac{\mathrm{w}_{\mathrm{F}} \int_{0}^{t} j_{t}(\theta, t) \mathrm{d} t}{\eta_{\mathrm{F}} \mathrm{F} \rho_{s}}
$$

where $\theta$ is the polar angle under polar coordinates; $u_{\text {red }}$ is the radius reduction; $\mathrm{w}_{\mathrm{F}}$ is the atomic weight of steel; $j_{t}$ is the total corrosion current density, which can be referred to literature [7]; $\eta_{F}$ is the valency of anodic reaction; F is the Faraday constant; $\rho_{s}$ is the density of steel reinforcement.

If we neglect the mutual effect on shear direction of rust product, for the sake of simplicity, the free expansion of corrosion product $u_{\exp }$ can be depicted by

$$
u_{\exp }(\theta, t)=(\kappa-1) u_{r e d}(\theta, t)
$$

where $\kappa$ is the expansion coefficient of steel reinforcement, which can be regarded as a constant according to literature [11]. 


\section{Conclusion}

This paper presents mathematical formulae to simulate the aggressive mass transport in concrete materials. A series of governing equations are given. The mechanism of corrosion process is discussed in an accurate method. An electrochemical corrosion process is described by mathematical equations.

\section{Acknowledgments}

This work was financially supported by the Natural Science Foundation of Shandong Province (No. ZR2018BEE044). This research was also supported by the Major Program of Mutual Foundation of Weihai City with Harbin Institute of Technology (Weihai), and Undergraduate Teaching Reform Research Project of Shandong Province (No. K2016M019).

\section{References}

[1] Guzmán S, Gálvez J C, Sancho J M. Modelling of corrosion-induced cover cracking in reinforced concrete by an embedded cohesive crack finite element[J]. Engineering Fracture Mechanics, 2012, 93(93):92-107.

[2] Ma Y, Guo Z, Wang L, et al. Experimental investigation of corrosion effect on bond behavior between reinforcing bar and concrete[J]. Construction \& Building Materials, 2017, 152:240-249.

[3] Zhao Y, Yu J, Wu Y, et al. Critical thickness of rust layer at inner and out surface cracking of concrete cover in reinforced concrete structures[J]. Corrosion Science, 2012, 59(6):316-323.

[4] Alonso C, Andrade C, Rodriguez J, et al. Factors controlling cracking of concrete affected by reinforcement corrosion[J]. Materials \& Structures, 1998, 31(7):435-441.

[5] Cao C, Cheung M M S, Chan B Y B. Modelling of interaction between corrosion-induced concrete cover crack and steel corrosion rate[J]. Corrosion Science, 2013, 69(1):97-109.

[6] Zhao Y, Hu B, Yu J, et al. Non-uniform distribution of rust layer around steel bar in concrete[J]. Corrosion Science, 2011, 53(12):4300-4308.

[7] Zhu X, Zi G. A 2D mechano-chemical model for the simulation of reinforcement corrosion and concrete damage[J]. Construction \& Building Materials, 2017, 137:330-344.

[8] Zhu X, Zi G, Cao Z, et al. Combined effect of carbonation and chloride ingress in concrete[J]. Construction \& Building Materials, 2016, 110:369-380.

[9]B. Hans (Ed.), Corrosion in Reinforced Concrete Structures, Woodhead Publishing Limited, England, 2005

[10] Cao C, Cheung M M S, Chan B Y B. Modelling of interaction between corrosion-induced concrete cover crack and steel corrosion rate[J]. Corrosion Science, 2013, 69(1):97-109.

[11] Suda K, Misra S, Motohashi K. Corrosion products of reinforcing bars embedded in concrete[J]. Corrosion Science, 1993, 35(5-8):1543-1549. 